\title{
The Scattering Matrix of a Graph
}

\author{
Hirobumi Mizuno \\ Iond University, Tokyo, Japan \\ Iwao Sato \\ Oyama National College of Technology, \\ Oyama, Tochigi 323-0806, Japan \\ isato@oyama-ct.ac.jp
}

Submitted: May 25, 2008; Accepted: Jul 16, 2008; Published: Jul 28, 2008

Mathematics Subject Classification: 05C50, 15A15

\begin{abstract}
Recently, Smilansky expressed the determinant of the bond scattering matrix of a graph by means of the determinant of its Laplacian. We present another proof for this Smilansky's formula by using some weighted zeta function of a graph. Furthermore, we reprove a weighted version of Smilansky's formula by Bass' method used in the determinant expression for the Ihara zeta function of a graph.
\end{abstract}

\section{Introduction}

Graphs treated here are finite. Let $G=(V(G), E(G))$ be a connected graph (possibly multiple edges and loops) with the set $V(G)$ of vertices and the set $E(G)$ of unoriented edges $u v$ joining two vertices $u$ and $v$. For $u v \in E(G)$, an arc $(u, v)$ is the oriented edge from $u$ to $v$. Set $R(G)=\{(u, v),(v, u) \mid u v \in E(G)\}$. For $b=(u, v) \in R(G)$, set $u=o(b)$ and $v=t(b)$. Furthermore, let $\hat{b}=(v, u)$ be the inverse of $b=(u, v)$.

A path $P$ of length $n$ in $G$ is a sequence $P=\left(b_{1}, \cdots, b_{n}\right)$ of $n$ arcs such that $b_{i} \in$ $R(G), t\left(b_{i}\right)=o\left(b_{i+1}\right)(1 \leq i \leq n-1)$, where indices are treated $\bmod n$. Set $|P|=n$, $o(P)=o\left(b_{1}\right)$ and $t(P)=t\left(b_{n}\right)$. Also, $P$ is called an $(o(P), t(P))$-path. We say that a path $P=\left(b_{1}, \cdots, b_{n}\right)$ has a backtracking or back-scatter if $\hat{b}_{i+1}=b_{i}$ for some $i(1 \leq i \leq n-1)$. A $(v, w)$-path is called a $v$-cycle (or $v$-closed path) if $v=w$. The inverse cycle of a cycle $C=\left(b_{1}, \cdots, b_{n}\right)$ is the cycle $\hat{C}=\left(\hat{b}_{n}, \cdots, \hat{b}_{1}\right)$.

We introduce an equivalence relation between cycles. Two cycles $C_{1}=\left(e_{1}, \cdots, e_{m}\right)$ and $C_{2}=\left(f_{1}, \cdots, f_{m}\right)$ are called equivalent if there exists $k$ such that $f_{j}=e_{j+k}$ for all $j$. The inverse cycle of $C$ is in general not equivalent to $C$. Let $[C]$ be the equivalence class which contains a cycle $C$. Let $B^{r}$ be the cycle obtained by going $r$ times around a cycle $B$. Such a cycle is called a power of $B$. A cycle $C$ is reduced if $C$ has no backtracking. 
Furthermore, a cycle $C$ is primitive if it is not a power of a strictly smaller cycle. Note that each equivalence class of primitive, reduced cycles of a graph $G$ corresponds to a unique conjugacy class of the fundamental group $\pi_{1}(G, u)$ of $G$ at a vertex $u$ of $G$. Furthermore, an equivalence class of primitive cycles of a graph $G$ is called a primitive periodic orbit of $G($ see $[13])$.

The Ihara zeta function of a graph $G$ is a function of a complex variable $t$ with $|t|$ sufficiently small, defined by

$$
\mathbf{Z}(G, t)=\mathbf{Z}_{G}(t)=\prod_{[p]}\left(1-t^{|p|}\right)^{-1},
$$

where $[p]$ runs over all primitive periodic orbits without back-scatter of $G($ see $[8])$.

Ihara zeta functions of graphs started from Ihara zeta functions of regular graphs by Ihara [8]. Originally, Ihara presented $p$-adic Selberg zeta functions of discrete groups, and showed that its reciprocal is a explicit polynomial. Serre [12] pointed out that the Ihara zeta function is the zeta function of the quotient $T / \Gamma$ (a finite regular graph) of the onedimensional Bruhat-Tits building $T$ (an infinite regular tree) associated with $G L\left(2, k_{p}\right)$.

A zeta function of a regular graph $G$ associated with a unitary representation of the fundamental group of $G$ was developed by Sunada $[15,16]$. Hashimoto [7] treated multivariable zeta functions of bipartite graphs. Bass [2] generalized Ihara's result on the zeta function of a regular graph to an irregular graph, and showed that its reciprocal is again a polynomial.

Theorem 1 (Bass) Let $G$ be a connected graph. Then the reciprocal of the zeta function of $G$ is given by

$$
\mathbf{Z}(G, t)^{-1}=\left(1-t^{2}\right)^{r-1} \operatorname{det}\left(\mathbf{I}-t \mathbf{C}(G)+t^{2}(\mathbf{D}-\mathbf{I})\right),
$$

where $r$ and $\mathbf{C}(G)$ are the Betti number and the adjacency matrix of $G$, respectively, and $\mathbf{D}=\left(d_{i j}\right)$ is the diagonal matrix with $d_{i i}=v_{i}=\operatorname{deg} u_{i}$ where $V(G)=\left\{u_{1}, \cdots, u_{n}\right\}$.

Various proofs of Bass' Theorem were given by Stark and Terras [14], Foata and Zeilberger [4], Kotani and Sunada [9].

Let $G$ be a connected graph. We say that a path $P=\left(b_{1}, \cdots, b_{n}\right)$ has a bump at $t\left(b_{i}\right)$ if $b_{i+1}=\hat{b}_{i}(1 \leq i \leq n)$. The cyclic bump count $\operatorname{cbc}(\pi)$ of a cycle $\pi=\left(\pi_{1}, \cdots, \pi_{n}\right)$ is

$$
\operatorname{cbc}(\pi)=\left|\left\{i=1, \cdots, n \mid \pi_{i}=\hat{\pi}_{i+1}\right\}\right|,
$$

where $\pi_{n+1}=\pi_{1}$. Then the Bartholdi zeta function of $G$ is a function of two complex variables $u, t$ with $|u|,|t|$ sufficiently small, defined by

$$
\zeta_{G}(u, t)=\zeta(G, u, t)=\prod_{[C]}\left(1-u^{c b c(C)} t^{|C|}\right)^{-1},
$$

where $[C]$ runs over all primitive periodic orbits of $G$ (see [1]). If $u=0$, then the Bartholdi zeta function of $G$ is the Ihara zeta function of $G$.

Bartholdi [1] gave a determinant expression of the Bartholdi zeta function of a graph. 
Theorem 2 (Bartholdi) Let $G$ be a connected graph with $n$ vertices and $m$ unoriented edges. Then the reciprocal of the Bartholdi zeta function of $G$ is given by

$$
\zeta(G, u, t)^{-1}=\left(1-(1-u)^{2} t^{2}\right)^{m-n} \operatorname{det}\left(\mathbf{I}-t \mathbf{C}(G)+(1-u)(\mathbf{D}-(1-u) \mathbf{I}) t^{2}\right) .
$$

In the case of $u=0$, Theorem 2 implies Theorem 1 .

Sato [11] defined a new zeta function of a graph by using not an infinite product but a determinant.

Let $G$ be a connected graph and $V(G)=\left\{u_{1}, \cdots, u_{n}\right\}$. Then we consider an $n \times n$ matrix $\tilde{\mathbf{C}}=\left(w_{i j}\right)_{1 \leq i, j \leq n}$ with $i j$ entry the complex variable $w_{i j}$ if $\left(u_{i}, u_{j}\right) \in R(G)$, and $w_{i j}=0$ otherwise. The matrix $\tilde{\mathbf{C}}=\tilde{\mathbf{C}}(G)$ is called the weighted matrix of $G$. For each path $P=\left(u_{i_{1}}, \cdots, u_{i_{r}}\right)$ of $G$, the norm $w(P)$ of $P$ is defined as follows: $w(P)=$ $w_{i_{1} i_{2}} w_{i_{2} i_{3}} \cdots w_{i_{r-1} i_{r}}$. Furthermore, let $w\left(u_{i}, u_{j}\right)=w_{i j}, u_{i}, u_{j} \in V(G)$ and $w(b)=w_{i j}, b=$ $\left(u_{i}, u_{j}\right) \in R(G)$.

Let $G$ be a connected graph with $n$ vertices and $m$ unoriented edges, and $\tilde{\mathbf{C}}=\tilde{\mathbf{C}}(G)$ a weighted matrix of $G$. Two $2 m \times 2 m$ matrices $\mathbf{B}=\mathbf{B}(G)=\left(\mathbf{B}_{e, f}\right)_{e, f \in R(G)}$ and $\mathbf{J}_{0}=$ $\mathbf{J}_{0}(G)=\left(\mathbf{J}_{e, f}\right)_{e, f \in R(G)}$ are defined as follows:

$$
\mathbf{B}_{e, f}=\left\{\begin{array}{ll}
w(f) & \text { if } t(e)=o(f), \\
0 & \text { otherwise }
\end{array}, \mathbf{J}_{e, f}= \begin{cases}1 & \text { if } f=\hat{e} \\
0 & \text { otherwise }\end{cases}\right.
$$

Then the zeta function of $G$ is defined by

$$
\mathbf{Z}_{1}(G, w, t)=\operatorname{det}\left(\mathbf{I}_{n}-t\left(\mathbf{B}-\mathbf{J}_{0}\right)\right)^{-1} .
$$

If $w(e)=1$ for any $e \in R(G)$, then the zeta function of $G$ is the Ihara zeta function of $G$.

Theorem 3 (Sato) Let $G$ be a connected graph, and let $\tilde{\mathbf{C}}=\tilde{\mathbf{C}}(G)$ be a weighted matrix of $G$. Then the reciprocal of the zeta function of $G$ is given by

$$
\mathbf{Z}_{1}(G, w, t)^{-1}=\left(1-t^{2}\right)^{m-n} \operatorname{det}\left(\mathbf{I}_{n}-t \tilde{\mathbf{C}}(G)+t^{2}\left(\tilde{\mathbf{D}}-\mathbf{I}_{n}\right)\right),
$$

where $n=|V(G)|, m=|E(G)|$ and $\tilde{\mathbf{D}}=\left(d_{i j}\right)$ is the diagonal matrix with $d_{i i}=$ $\sum_{o(b)=u_{i}} w(e), V(G)=\left\{u_{1}, \cdots, u_{n}\right\}$.

The spectral determinant of the Laplacian on a quantum graph is closely related to the Ihara zeta function of a graph(see $[3,5,6,13])$.

Smilansky [13] considered spectral zeta functions and trace formulas for (discrete) Laplacians on ordinary graphs, and expressed some determinant on the bond scattering matrix of a graph $G$ by using the characteristic polynomial of its Laplacian.

Let $G$ be a connected graph with $n$ vertices and $m$ edges, $V(G)=\left\{u_{1}, \ldots, u_{n}\right\}$ and $R(G)=\left\{b_{1}, \ldots, b_{m}, b_{m+1}, \ldots, b_{2 m}\right\}$ such that $b_{m+j}=\hat{b}_{j}(1 \leq j \leq m)$.

The Laplacian (matrix) $\mathbf{L}=\mathbf{L}(G)$ of $G$ is defined by

$$
\mathbf{L}=\mathbf{L}(G)=-\mathbf{C}(G)+\mathbf{D} .
$$


Let $\lambda$ be a eigenvalue of $\mathbf{L}$ and $\psi=\left(\psi_{1}, \ldots, \psi_{n}\right)$ the eigenvector corresponding to $\lambda$. For each $\operatorname{arc} b=\left(u_{j}, u_{l}\right)$, one associates a bond wave function

$$
\psi_{b}(x)=a_{b} \mathrm{e}^{i \pi x / 4}+a_{\hat{b}} \mathrm{e}^{-i \pi x / 4}, x= \pm 1
$$

under the condition

$$
\psi_{b}(1)=\psi_{j}, \psi_{b}(-1)=\psi_{l} .
$$

We consider the following three conditions:

1. uniqueness: The value of the eigenvector at the vertex $u_{j}, \psi_{j}$, computed in the terms of the bond wave functions is the same for all the arcs emanating from $u_{j}$.

2. $\psi$ is an eigenvector of $\mathbf{L}$;

3. consistency: The linear relation between the incoming and the outgoing coefficients (1) must be satisfied simultaneously at all vertices.

By the uniqueness, we have

$$
a_{b_{1}} \mathrm{e}^{i \pi / 4}+a_{\hat{b}_{1}} \mathrm{e}^{-i \pi / 4}=a_{b_{2}} \mathrm{e}^{i \pi / 4}+a_{\hat{b}_{2}} \mathrm{e}^{-i \pi / 4}=\cdots=a_{b_{v_{j}}} \mathrm{e}^{i \pi / 4}+a_{\hat{b}_{v_{j}}} \mathrm{e}^{-i \pi / 4},
$$

where $b_{1}, b_{2}, \ldots, b_{v_{j}}$ are arcs emanating from $u_{j}$, and $v_{j}=\operatorname{deg} u_{j}, i=\sqrt{-1}$.

By the condition 2, we have

$$
-\sum_{k=1}^{v_{j}}\left(a_{b_{k}} \mathrm{e}^{-i \pi / 4}+a_{\hat{b}_{k}} \mathrm{e}^{i \pi / 4}\right)=\left(\lambda-v_{j}\right) \frac{1}{v_{j}} \sum_{k=1}^{v_{j}}\left(a_{b_{k}} \mathrm{e}^{i \pi / 4}+a_{\hat{b}_{k}} \mathrm{e}^{-i \pi / 4}\right) .
$$

Thus, for each $\operatorname{arc} b$ with $o(b)=u_{j}$,

$$
a_{b}=\sum_{t(c)=u_{j}} \sigma_{b, c}^{\left(u_{j}\right)}(\lambda) a_{c}
$$

where

$$
\sigma_{b, c}^{\left(u_{j}\right)}(\lambda)=i\left(\delta_{\hat{b}, c}-\frac{2}{v_{j}} \frac{1}{1-i\left(1-\lambda / v_{j}\right)}\right),
$$

and $\delta_{\hat{b}, c}$ is the Kronecker delta. The bond scattering matrix $\mathbf{U}(\lambda)=\left(U_{e f}\right)_{e, f \in R(G)}$ of $G$ is defined by

$$
U_{e f}= \begin{cases}\sigma_{e, f}^{(t(f))} & \text { if } t(f)=o(e) \\ 0 & \text { otherwise. }\end{cases}
$$

By the consistency, we have

$$
\mathbf{U}(\lambda) \mathbf{a}=\mathbf{a},
$$

where $\mathbf{a}={ }^{t}\left(a_{b_{1}}, a_{b_{2}}, \ldots, a_{b_{2 m}}\right)$. This holds if and only if

$$
\operatorname{det}\left(\mathbf{I}_{2 m}-\mathbf{U}(\lambda)\right)=0
$$


Theorem 4 (Smilansky) Let $G$ be a connected graph with $n$ vertices and $m$ edges. Then the characteristic polynomial of the bond scattering matrix of $G$ is given by

$$
\operatorname{det}\left(\mathbf{I}_{2 m}-\mathbf{U}(\lambda)\right)=\frac{2^{m} i^{n} \operatorname{det}\left(\lambda \mathbf{I}_{n}+\mathbf{C}(G)-\mathbf{D}\right)}{\prod_{j=1}^{n}\left(v_{j}-i v_{j}+\lambda i\right)}=\prod_{[p]}\left(1-a_{p}(\lambda)\right),
$$

where $[p]$ runs over all primitive periodic orbits of $G$, and

$$
a_{p}(\lambda)=\sigma_{b_{1}, b_{n}}^{\left(t\left(b_{n}\right)\right)} \sigma_{b_{n}, b_{n-1}}^{\left(t\left(b_{n-1}\right)\right)} \cdots \sigma_{b_{2}, b_{1}}^{\left(t\left(b_{1}\right)\right)}, p=\left(b_{1}, b_{2}, \ldots, b_{n}\right) .
$$

In this paper, we reprove Smilansky's formula for the characteristic polynomial of the bond scattering matrix of a graph and its weighted version by using some zeta functions of a graph. In Section 2, we consider a new zeta function of a graph $G$, and present another proof of Smilansky's formula for some determinant on the bond scattering matrix of a graph by means of the Laplacian of $G$. Furthermore, we give Smilansky's formula for the case of a regular graph by using Bartholdi zeta function of a graph. In Section 3, we present a decomposition formula for some determinant on the bond scattering matrix of a semiregular bipartite graph. In Section 4, we give another proof for a weighted version of the above Smilansky's formula by Bass' method used in the determinant expression for the Ihara zeta function of a graph. In Section 5, we express a new zeta function of a graph by using the Euler product.

\section{The scattering matrix of a graph}

We present a proof of Theorem 4 by using Theorem 3, which is different from a proof in [13].

Theorem 5 (Smilansky) Let $G$ be a connected graph with $n$ vertices and $m$ edges. Then, for the bond scattering matrix of $G$,

$$
\operatorname{det}\left(\mathbf{I}_{2 m}-\mathbf{U}(\lambda)\right)=\frac{2^{m} i^{n} \operatorname{det}\left(\lambda \mathbf{I}_{n}+\mathbf{C}(G)-\mathbf{D}\right)}{\prod_{j=1}^{n}\left(v_{j}-i v_{j}+\lambda i\right)} .
$$

Proof. Let $G$ be a connected graph with $n$ vertices and $m$ edges, $V(G)=\left\{u_{1}, \cdots, u_{n}\right\}$ and $R(G)=\left\{b_{1}, \ldots, b_{m}, \hat{b}_{1}, \ldots, \hat{b}_{m}\right\}$. Set $v_{j}=\operatorname{deg} u_{j}$ and

$$
x_{j}=x_{u_{j}}=\frac{2}{v_{j}} \frac{1}{1-i\left(1-\lambda / v_{j}\right)}
$$

for each $j=1, \ldots, n$. Then we consider a $2 m \times 2 m$ matrix $\mathbf{B}=\left(B_{e f}\right)_{e, f \in R(G)}$ given by

$$
B_{e f}= \begin{cases}x_{o(f)} & \text { if } t(e)=o(f) \\ 0 & \text { otherwise }\end{cases}
$$


By Theorem 3, we have

$$
\operatorname{det}\left(\mathbf{I}_{2 m}-u\left(\mathbf{B}-\mathbf{J}_{0}\right)\right)=\left(1-u^{2}\right)^{m-n} \operatorname{det}\left(\mathbf{I}_{n}-u \mathbf{W}_{x}(G)+u^{2}\left(\mathbf{D}_{x}-\mathbf{I}_{n}\right)\right),
$$

where $\mathbf{W}_{x}(G)=\left(w_{j k}\right)$ and $\mathbf{D}_{x}=\left(d_{j k}\right)$ are given as follows:

$$
w_{j k}=\left\{\begin{array}{ll}
x_{j} & \text { if }\left(u_{j}, u_{k}\right) \in R(G), \\
0 & \text { otherwise }
\end{array}, d_{j k}= \begin{cases}v_{j} x_{j} & \text { if } j=k \\
0 & \text { otherwise }\end{cases}\right.
$$

Thus,

$$
\operatorname{det}\left(\mathbf{I}_{2 m}-u\left({ }^{t} \mathbf{B}-{ }^{t} \mathbf{J}_{0}\right)\right)=\left(1-u^{2}\right)^{m-n} \operatorname{det}\left(\mathbf{I}_{n}-u \mathbf{W}_{x}(G)+u^{2}\left(\mathbf{D}_{x}-\mathbf{I}_{n}\right)\right),
$$

where ${ }^{t} \mathbf{B}$ is the transpose of $\mathbf{B}$. Note that

$$
v_{j} x_{j}=\frac{2}{1-i\left(1-\lambda / v_{j}\right)}(1 \leq j \leq n) .
$$

But, since

$$
i \mathbf{U}(\lambda)+\mathbf{J}_{0}={ }^{t} \mathbf{B}
$$

we have

$$
{ }^{t} \mathbf{B}-{ }^{t} \mathbf{J}_{0}=i \mathbf{U}(\lambda) .
$$

Substituting $u=-i$ in (2), we obtain

$$
\operatorname{det}\left(\mathbf{I}_{2 m}-\mathbf{U}(\lambda)\right)=2^{m-n} \operatorname{det}\left(\mathbf{I}_{n}+i \mathbf{W}_{x}(G)-\left(\mathbf{D}_{x}-\mathbf{I}_{n}\right)\right) .
$$

Now, we have

$$
\mathbf{W}_{x}(G)=\left[\begin{array}{ccc}
x_{1} & & 0 \\
& \ddots & \\
0 & & x_{n}
\end{array}\right] \mathbf{C}(G)
$$

and

$$
\mathbf{D}_{x}=\left[\begin{array}{ccc}
x_{1} & & 0 \\
& \ddots & \\
0 & & x_{n}
\end{array}\right] \mathbf{D} .
$$

Let

$$
\mathbf{X}=\left[\begin{array}{ccc}
x_{1} & & 0 \\
& \ddots & \\
0 & & x_{n}
\end{array}\right]
$$

Then it follows that

$$
\begin{gathered}
\operatorname{det}\left(\mathbf{I}_{2 m}-\mathbf{U}(\lambda)\right)=2^{m-n} \operatorname{det}\left(2 \mathbf{I}_{n}+i \mathbf{X C}(G)-\mathbf{X D}\right) \\
=2^{m-n} i^{n} \operatorname{det} \mathbf{X} \operatorname{det}\left(-2 i \mathbf{X}^{-1}+\mathbf{C}(G)+i \mathbf{D}\right)=\frac{2^{m} i^{n} \operatorname{det}\left(-2 i \mathbf{X}^{-1}+\mathbf{C}(G)+i \mathbf{D}\right)}{\prod_{j=1}^{n}\left(v_{j}-i v_{j}+\lambda i\right)} .
\end{gathered}
$$


Since $2 x_{j}^{-1}=v_{j}-i v_{j}+\lambda i$, we have

$$
-2 i \mathbf{X}^{-1}=-i(1-i) \mathbf{D}+\lambda \mathbf{I}_{n}
$$

and so

$$
-2 i \mathbf{X}^{-1}+\mathbf{C}(G)+i \mathbf{D}=\lambda \mathbf{I}_{n}+\mathbf{C}(G)-\mathbf{D}
$$

Hence

$$
\operatorname{det}\left(\mathbf{I}_{2 m}-\mathbf{U}(\lambda)\right)=\frac{2^{m} i^{n} \operatorname{det}\left(\lambda \mathbf{I}_{n}+\mathbf{C}(G)-\mathbf{D}\right)}{\prod_{j=1}^{n}\left(v_{j}-i v_{j}+\lambda i\right)}
$$

Q.E.D.

We present some determinant on the bond scattering matrix of a regular graph $G$ by using the Bartholdi zeta function of $G$.

Corollary 1 (Smilansky) Let $G$ be an r-reguar graph with $n$ vertices and $m$ edges. Then, for the bond scattering matrix of $G$,

$$
\operatorname{det}\left(\mathbf{I}_{2 m}-\mathbf{U}(\lambda)\right)=2^{m} i^{n}(r-i r+\lambda i)^{-n} \operatorname{det}\left(\lambda \mathbf{I}_{n}+\mathbf{C}(G)-r \mathbf{I}_{n}\right) .
$$

Proof. Let $G$ be an $r$-regular graph with $n$ vertices and $m$ edges, $V(G)=\left\{u_{1}, \cdots, u_{n}\right\}$ and $R(G)=\left\{b_{1}, \ldots, b_{m}, \hat{b}_{1}, \ldots, \hat{b}_{m}\right\}$. Then we have

$$
x=x_{j}=x_{u_{j}}=\frac{2}{r} \frac{1}{1-i(1-\lambda / r)}
$$

for each $j=1, \ldots, n$. Thus, each $\sigma_{b, c}^{(t(c))}(\lambda)$ in (1) are given by

$$
\sigma_{b, c}^{(t(c))}= \begin{cases}-i x & \text { if } t(c)=o(b) \\ i(1-x) & \text { if } c=\hat{b} \\ 0 & \text { otherwise. }\end{cases}
$$

By Theorem 4, we have

$$
\operatorname{det}\left(\mathbf{I}_{2 m}-\mathbf{U}(\lambda)\right)^{-1}=\prod_{[p]}\left(1-a_{p}(\lambda)\right)^{-1},
$$

where $[p]$ runs over all primitive periodic orbits of $G$. Since

$$
a_{p}(\lambda)=\sigma_{b_{1}, b_{n}}^{\left(t\left(b_{n}\right)\right)} \sigma_{b_{n}, b_{n-1}}^{\left(t\left(b_{n-1}\right)\right)} \cdots \sigma_{b_{2}, b_{1}}^{\left(t\left(b_{1}\right)\right)}, p=\left(b_{1}, b_{2}, \ldots, b_{n}\right)
$$

we have

$$
\begin{aligned}
\operatorname{det}\left(\mathbf{I}_{2 m}-\mathbf{U}(\lambda)\right) & =\prod_{[p]}\left(1-(i(1-x))^{c b c(p)}(-i x)^{|p|-c b c(p)}\right)^{-1} \\
& =\prod_{[p]}\left(1-\left(\frac{i(1-x)}{-i x}\right)^{c b c(p)}(-i x)^{|p|}\right)^{-1}
\end{aligned}
$$


Now, let

$$
u=\frac{i(1-x)}{-i x}, t=-i x .
$$

By Theorem 2, since $u=1+i / t$, we have

$$
\begin{aligned}
\operatorname{det}\left(\mathbf{I}_{2 m}-\mathbf{U}(\lambda)\right) & =\left(1-(1-u)^{2} t^{2}\right)^{m-n} \operatorname{det}\left(\mathbf{I}_{n}-t \mathbf{C}(G)+(1-u) t^{2}\left(r \mathbf{I}_{n}-(1-u) \mathbf{I}_{n}\right)\right) \\
& =2^{m-n} \operatorname{det}\left(\mathbf{I}_{n}-t \mathbf{C}(G)-i(r t+i) \mathbf{I}_{n}\right) \\
& =2^{m-n} \operatorname{det}\left(2 \mathbf{I}_{n}-t\left(\mathbf{C}(G)+i r \mathbf{I}_{n}\right)\right) \\
& =2^{m-n}(-t)^{n} \operatorname{det}\left(-2 / t \mathbf{I}_{n}+\mathbf{C}(G)+i r \mathbf{I}_{n}\right)
\end{aligned}
$$

Since

$$
-\frac{2}{t}=-i(r-r i+\lambda i)
$$

we have

$$
\operatorname{det}\left(\mathbf{I}_{2 m}-\mathbf{U}(\lambda)\right)=2^{m-n} i^{n}(r-r i+\lambda)^{-n} \operatorname{det}\left(\lambda \mathbf{I}_{n}+\mathbf{C}(G)-r \mathbf{I}_{n}\right) .
$$

Q.E.D.

\section{The scattering matrix of a semiregular bipartite graph}

We present a decomposition formula for some determinant on the scattering matrix of a semiregular bipartite graph.

A graph $G$ is called bipartite, denoted by $G=\left(V_{1}, V_{2}\right)$ if there exists a partition $V(G)=V_{1} \cup V_{2}$ of $V(G)$ such that $u v \in E(G)$ if and only if $u \in V_{1}$ and $v \in V_{2}$. A bipartite graph $G=\left(V_{1}, V_{2}\right)$ is called $\left(q_{1}+1, q_{2}+1\right)$-semiregular if $\operatorname{deg}_{G} v=q_{i}+1$ for each $v \in V_{i}(i=1,2)$. For a $\left(q_{1}+1, q_{2}+1\right)$-semiregular bipartite graph $G=\left(V_{1}, V_{2}\right)$, let $G^{[i]}$ be the graph with vertex set $V_{i}$ and an edge between two vertices in $G^{[i]}$ if there is a path of length two between them in $G$ for $i=1,2$. Then $G^{[1]}$ is $\left(q_{1}+1\right) q_{2}$-regular, and $G^{[2]}$ is $\left(q_{2}+1\right) q_{1}$-regular.

By Theorem 5, we obtain the following result.

Theorem 6 Let $G=\left(V_{1}, V_{2}\right)$ be a connected $\left(q_{1}+1, q_{2}+1\right)$-semiregular bipartite graph with $\nu$ vertices and $\epsilon$ edges. Set $\left|V_{1}\right|=n,\left|V_{2}\right|=m(n \leq m)$. Then

$\operatorname{det}\left(\mathbf{I}_{2 \epsilon}-\mathbf{U}(\lambda)\right)=2^{m} i^{n}\left(\lambda-q_{2}-1\right)^{m-n} \frac{\prod_{j=1}^{n}\left(\lambda^{2}-\left(q_{1}+q_{2}-2\right) \lambda+\left(q_{1}+1\right)\left(q_{2}+1\right)-\lambda_{j}^{2}\right)}{\left(\left(q_{1}+1\right)(1-i)+\lambda i\right)^{n}\left(\left(q_{2}+1\right)(1-i)+\lambda i\right)^{m}}$.

where $\operatorname{Spec}(G)=\left\{ \pm \lambda_{1}, \cdots, \pm \lambda_{n}, 0, \cdots, 0\right\}$. 
Proof. The argument is an analogue of Hashimoto's method [7].

By Theorem 5, we have

$$
\operatorname{det}\left(\mathbf{I}_{2 \epsilon}-\mathbf{U}(\lambda)\right)=\frac{2^{\epsilon} i^{\nu} \operatorname{det}\left(\lambda \mathbf{I}_{\nu}+\mathbf{C}(G)-\mathbf{D}\right)}{\left(\left(q_{1}+1\right)(1-i)+\lambda i\right)^{n}\left(\left(q_{2}+1\right)(1-i)+\lambda i\right)^{m}} .
$$

Let $V_{1}=\left\{u_{1}, \cdots, u_{n}\right\}$ and $V_{2}=\left\{s_{1}, \cdots, s_{m}\right\}$. Arrange vertices of $G$ as follows: $u_{1}, \cdots, u_{n} ; v_{1}, \cdots, v_{m}$. We consider the matrix $\mathbf{C}(G)$ under this order. Then, with the definition, we can see that

$$
\mathbf{C}(G)=\left[\begin{array}{cc}
\mathbf{0} & \mathbf{B} \\
{ }^{t} \mathbf{B} & \mathbf{0}
\end{array}\right]
$$

Since $\mathbf{C}(G)$ is symmetric, there exists a orthogonal matrix $\mathbf{U} \in U(m)$ such that

$$
\mathbf{B U}=\left[\begin{array}{ll}
\mathbf{C} & \mathbf{0}
\end{array}\right]=\left[\begin{array}{cccccc}
\mu_{1} & & 0 & 0 & \cdots & 0 \\
& \ddots & & \vdots & & \vdots \\
\star & & \mu_{n} & 0 & \cdots & 0
\end{array}\right] \text {. }
$$

Now, let

$$
\mathbf{P}=\left[\begin{array}{cc}
\mathbf{I}_{n} & \mathbf{0} \\
\mathbf{0} & \mathbf{U}
\end{array}\right]
$$

Then we have

$$
{ }^{t} \mathbf{P C}(G) \mathbf{P}=\left[\begin{array}{ccc}
\mathbf{0} & \mathbf{F} & \mathbf{0} \\
{ }^{t} \mathbf{F} & \mathbf{0} & \mathbf{0} \\
\mathbf{0} & \mathbf{0} & \mathbf{0}
\end{array}\right],
$$

where ${ }^{t} \mathbf{F}$ is the transpose of $\mathbf{F}$. Furthermore, we have

$$
{ }^{t} \mathbf{P D P}=\mathbf{D} .
$$

Thus,

$$
\begin{aligned}
\operatorname{det}\left(\mathbf{I}_{2 \epsilon}-\mathbf{U}(\lambda)\right) \\
=\frac{2^{m} i^{n}\left(\lambda-q_{2}-1\right)^{m-n}}{\left(\left(q_{1}+1\right)(1-i)+\lambda i\right)^{n}\left(\left(q_{2}+1\right)(1-i)+\lambda i\right)^{m}} \operatorname{det}\left[\begin{array}{cc}
\left(\lambda-q_{1}-1\right) \mathbf{I}_{n} & -\mathbf{F} \\
-{ }^{t} \mathbf{F} & \left(\lambda-q_{2}-1\right) \mathbf{I}_{n}
\end{array}\right] \\
=\frac{2^{m} i^{n}\left(\lambda-q_{2}-1\right)^{m-n}}{\left(\left(q_{1}+1\right)(1-i)+\lambda i\right)^{n}\left(\left(q_{2}+1\right)(1-i)+\lambda i\right)^{m}} \\
\quad \times \operatorname{det}\left[\begin{array}{c}
\left(\lambda-q_{1}-1\right) \mathbf{I}_{n} \\
-{ }^{t} \mathbf{F} \quad\left(\lambda-q_{2}-1\right) \mathbf{I}_{n}-\left(\lambda-q_{1}-1\right)^{-1 t} \mathbf{F F}
\end{array}\right] \\
=\frac{2^{m} i^{n}\left(\lambda-q_{2}-1\right)^{m-n}}{\left(\left(q_{1}+1\right)(1-i)+\lambda i\right)^{n}\left(\left(q_{2}+1\right)(1-i)+\lambda i\right)^{m}} \operatorname{det}\left(\left(\lambda-q_{1}-1\right)\left(\lambda-q_{2}-1\right) \mathbf{I}_{n}-{ }^{t} \mathbf{F F}\right) .
\end{aligned}
$$

Since $\mathbf{C}(G)$ is symmetric, ${ }^{t} \mathbf{F F}$ is Hermitian and positive definite, i.e., the eigenvalues of ${ }^{t} \mathbf{F F}$ are of form:

$$
\lambda_{1}^{2}, \cdots, \lambda_{n}^{2}\left(\lambda_{1}, \cdots, \lambda_{n} \geq 0\right) .
$$


Therefore it follows that

$\operatorname{det}\left(\mathbf{I}_{2 \epsilon}-\mathbf{U}(\lambda)\right)=2^{m} i^{n}\left(\lambda-q_{2}-1\right)^{m-n} \frac{\prod_{j=1}^{n}\left(\lambda^{2}-\left(q_{1}+q_{2}-2\right) \lambda+\left(q_{1}+1\right)\left(q_{2}+1\right)-\lambda_{j}^{2}\right)}{\left(\left(q_{1}+1\right)(1-i)+\lambda i\right)^{n}\left(\left(q_{2}+1\right)(1-i)+\lambda i\right)^{m}}$.

But, we have

$$
\operatorname{det}(\lambda \mathbf{I}-\mathbf{C}(G))=\lambda^{(m-n)} \operatorname{det}\left(\lambda^{2} \mathbf{I}-{ }^{t} \mathbf{F F}\right),
$$

and so

$$
\operatorname{Spec}(G)=\left\{ \pm \lambda_{1}, \cdots, \pm \lambda_{n}, 0, \cdots, 0\right\} .
$$

Therefore, the result follows. Q.E.D.

\section{A weighted version of the scattering matrix of a graph}

Let $G$ be a connected graph with $n$ vertices and $m$ unoriented edges, and $\tilde{\mathbf{C}}=\tilde{\mathbf{C}}(G)$ a symmertic weighted matrix of $G$ with all nonnegative elements. Then $\tilde{\mathbf{C}}(G)$ is called a non-negative symmetric weighted matrix of $G$. Set $V(G)=\left\{u_{1}, \cdots, u_{n}\right\}, R(G)=$ $\left\{b_{1}, \ldots, b_{m}, \hat{b}_{1}, \ldots, \hat{b}_{m}\right\}$. and

$$
v_{j}=\sum_{o(b)=u_{j}} w(b) \text { for } j=1, \ldots, n .
$$

Smilansky [13] considered a weighted version of the characteristic polynomial of the bond scattering matrix of a regular graph $G$, and expressed it by using the characteristic polynomial of its weighted Laplacian of $G$.

The weighted bond scattering matrix $\mathbf{U}(\lambda)=\left(U_{e f}\right)_{e, f \in R(G)}$ of $G$ is defined by

$$
U_{e f}= \begin{cases}i\left(\delta_{\hat{e}, f}-x_{t(f)} \sqrt{w(e)} \sqrt{w(f)}\right) & \text { if } t(f)=o(e), \\ 0 & \text { otherwise }\end{cases}
$$

where

$$
x_{j}=x_{u_{j}}=\frac{2}{v_{j}} \frac{1}{1-i\left(1-\lambda / v_{j}\right)}
$$

for each $j=1, \ldots, n$.

Smilansky [13] stated a formula for some determinant on the weighted scattering matrix of a graph $G$ without a proof.

Theorem 7 (Smilansky) Let $G$ be a connected graph with $n$ vertices and $m$ unoriented edges and $\tilde{\mathbf{C}}(G)$ a non-negative symmetric weighted matrix of $G$. Then, for the weighted scattering matrix of $G$,

$$
\operatorname{det}\left(\mathbf{I}_{2 m}-\mathbf{U}(\lambda)\right)=\frac{2^{m} i^{n} \operatorname{det}\left(\lambda \mathbf{I}_{n}+\tilde{\mathbf{C}}(G)-\tilde{\mathbf{D}}\right)}{\prod_{j=1}^{n}\left(v_{j}-i v_{j}+\lambda i\right)} .
$$


Proof. The argument is an analogue of Bass' method [2].

Let $\rho$ be a unitary representation of $\Gamma$, and $d$ the degree of $\rho$. Furthermore, let $V(G)=$ $\left\{u_{1}, \cdots, u_{n}\right\}$ and $R(G)=\left\{b_{1}, \cdots, b_{m}, b_{m+1}, \cdots, b_{2 m}\right\}$ such that $b_{m+i}=\hat{b}_{i}(1 \leq i \leq m)$. Let $\mathbf{K}=\left(\mathbf{K}_{i, j}\right)_{1 \leq i \leq 2 l ; 1 \leq j \leq n}$ be the $2 l \times n$ matrix defined as follows:

$$
\mathbf{K}_{i, j}:= \begin{cases}\sqrt{w\left(b_{i}\right)} & \text { if } t\left(b_{i}\right)=u_{j} \\ 0 & \text { otherwise. }\end{cases}
$$

Next we define two $2 m \times n$ matrices $\mathbf{L}=\left(\mathbf{L}_{i, j}\right)_{1 \leq i \leq 2 m ; 1 \leq j \leq n}$ and $\mathbf{H}=\left(\mathbf{H}_{i, j}\right)_{1 \leq i \leq 2 m ; 1 \leq j \leq n}$ as follows:

$$
\mathbf{L}_{i, j}:=\left\{\begin{array}{ll}
\sqrt{w\left(b_{i}\right)} x_{u_{j}} & \text { if } o\left(b_{i}\right)=u_{j}, \\
0 & \text { otherwise. }
\end{array}, \mathbf{H}_{i, j}:= \begin{cases}\sqrt{w\left(b_{i}\right)} & \text { if } o\left(b_{i}\right)=u_{j} \\
0 & \text { otherwise. }\end{cases}\right.
$$

Note that

$$
\mathbf{L}=\mathbf{H}\left[\begin{array}{ccc}
x_{u_{1}} & & 0 \\
& \ddots & \\
0 & & x_{u_{n}}
\end{array}\right]=\mathbf{H X}
$$

Then we have

$$
\mathbf{L}^{t} \mathbf{K}={ }^{t} \mathbf{B} .
$$

and

$$
{ }^{t} \mathbf{H K}=\tilde{\mathbf{C}}(G),
$$

where two matrices $\mathbf{B}=\left(B_{e f}\right)_{e, f \in R(G)}$ and $\tilde{\mathbf{C}}(G)=\left(w_{u s}\right)_{u, s \in V(G)}$ are given by

$$
B_{e f}:=\left\{\begin{array}{ll}
x_{t(e)} \sqrt{w(e) w(f)} & \text { if } t(e)=o(f), \\
0 & \text { otherwise. }
\end{array}, w_{u v}:= \begin{cases}w(u, s) & \text { if }(u, s) \in R(G), \\
0 & \text { otherwise. }\end{cases}\right.
$$

Furthermore,

$$
{ }^{t} \mathbf{H H}=\tilde{\mathbf{D}} .
$$

Next, we have

$$
{ }^{t} \mathbf{K L}={ }^{t} \mathbf{W}_{x}(G)
$$

and

$$
{ }^{t} \mathbf{H L}=\mathbf{D}_{x},
$$

where two matrices $\mathbf{W}_{x}=\left(\left(w_{x}\right)_{u s}\right)_{u, s \in V(G)}$ and $\mathbf{D}_{x}=\left(d_{u s}\right)_{u, s \in V(G)}$ are given by

$$
\left(w_{x}\right)_{u s}:=\left\{\begin{array}{ll}
w(u, s) x_{u} & \text { if }(u, s) \in R(G), \\
0 & \text { otherwise. }
\end{array}, d_{u s}:= \begin{cases}v_{u} x_{u} & \text { if } u=s, \\
0 & \text { otherwise. }\end{cases}\right.
$$

Now, let

$$
\mathbf{J}=\left[\begin{array}{cc}
\mathbf{0} & w\left(b_{1}\right) x_{o\left(\left(\hat{b}_{1}\right)\right.} \oplus \cdots \oplus w\left(b_{m}\right) x_{o\left(\hat{b}_{m}\right)} \\
w\left(b_{1}\right) x_{o\left(b_{1}\right)} \oplus \cdots \oplus w\left(b_{m}\right) x_{o\left(b_{m}\right)} & \mathbf{0}
\end{array}\right]
$$


and

$$
\mathbf{T}=\mathbf{B}-\mathbf{J}
$$

Then we have

$$
\mathbf{L}^{t} \mathbf{H}={ }^{t} \mathbf{T}^{t} \mathbf{J}_{0}+\left(w\left(b_{1}\right) x_{o\left(b_{1}\right)} \oplus \cdots \oplus w\left(\hat{b}_{m}\right) x_{o\left(\hat{b}_{m}\right)}\right) .
$$

We introduce two $(2 m+n) \times(2 m+n)$ matrices as follows:

$$
\mathbf{P}=\left[\begin{array}{cc}
\left(1-u^{2}\right) \mathbf{I}_{n} & -{ }^{t} \mathbf{K}+u{ }^{t} \mathbf{H} \\
\mathbf{0} & \mathbf{I}_{2 m}
\end{array}\right], \mathbf{Q}=\left[\begin{array}{cc}
\mathbf{I}_{n} & { }^{t} \mathbf{K}-u{ }^{t} \mathbf{H} \\
u \mathbf{L} & \left(1-u^{2}\right) \mathbf{I}_{2 m}
\end{array}\right]
$$

By (8) and (9), we have

$$
\begin{aligned}
\mathbf{P Q} & =\left[\begin{array}{cc}
\left(1-u^{2}\right) \mathbf{I}_{n}-u{ }^{t} \mathbf{K} \mathbf{L}+u^{2}{ }^{t} \mathbf{H L} & \mathbf{0} \\
u \mathbf{L} & \left(1-u^{2}\right) \mathbf{I}_{2 m}
\end{array}\right] \\
& =\left[\begin{array}{cc}
\left(1-u^{2}\right) \mathbf{I}_{n}-u{ }^{t} \mathbf{W}_{x}(G)+u^{2} \mathbf{D}_{x} & \\
u \mathbf{L} & \left(1-u^{2}\right) \mathbf{I}_{2 m}
\end{array}\right] .
\end{aligned}
$$

By (5) and (10),

$$
\mathbf{Q P}=\left[\begin{array}{cc}
\left(1-u^{2}\right) \mathbf{I}_{n} & \mathbf{0} \\
u\left(1-u^{2}\right) \mathbf{L} & -u \mathbf{L}^{t} \mathbf{K}+u^{2} \mathbf{L}^{t} \mathbf{H}+\left(1-u^{2}\right) \mathbf{I}_{2 m}
\end{array}\right] .
$$

Since

$$
w\left(b_{1}\right) x_{o\left(b_{1}\right)} \oplus \cdots \oplus w\left(\hat{b}_{m}\right) x_{o\left(\hat{b}_{m}\right)}={ }^{t} \mathbf{J}^{t} \mathbf{J}_{0}
$$

and $\left({ }^{t} \mathbf{J}_{0}\right)^{2}=\mathbf{I}_{2 m}$, we have

$$
\begin{aligned}
& -u \mathbf{L}^{t} \mathbf{K}+u^{2} \mathbf{L}^{t} \mathbf{H}+\left(1-u^{2}\right) \mathbf{I}_{2 m} \\
= & \mathbf{I}_{2 m}-u\left({ }^{t} \mathbf{T}+{ }^{t} \mathbf{J}\right)+u^{2}\left({ }^{t} \mathbf{T}^{t} \mathbf{J}_{0}+{ }^{t} \mathbf{J}^{t} \mathbf{J}_{0}-{ }^{t} \mathbf{J}_{0}{ }^{t} \mathbf{J}_{0}\right) \\
= & \left(\mathbf{I}_{2 m}-u\left({ }^{t} \mathbf{T}+{ }^{t} \mathbf{J}-{ }^{t} \mathbf{J}_{0}\right)\right)\left(\mathbf{I}_{2 m}-u{ }^{t} \mathbf{J}_{0}\right) .
\end{aligned}
$$

Thus,

$$
\mathbf{Q P}=\left[\begin{array}{cc}
\left(1-u^{2}\right) \mathbf{I}_{n} & \mathbf{0} \\
u\left(1-u^{2}\right) \mathbf{L} & \left(\mathbf{I}_{2 m}-u\left({ }^{t} \mathbf{T}+{ }^{t} \mathbf{J}-{ }^{t} \mathbf{J}_{0}\right)\right)\left(\mathbf{I}_{2 m}-u^{t} \mathbf{J}_{0}\right)
\end{array}\right] .
$$

Since $\operatorname{det}(\mathbf{P Q})=\operatorname{det}(\mathbf{Q P})$, we have

$$
\begin{gathered}
\left(1-u^{2}\right)^{2 m} \operatorname{det}\left(\mathbf{I}_{n}-u{ }^{t} \mathbf{W}_{x}(G)+\left(\mathbf{D}_{x}-\mathbf{I}_{n}\right) u^{2}\right) \\
=\left(1-u^{2}\right)^{n} \operatorname{det}\left(\mathbf{I}_{2 m}-u\left({ }^{t} \mathbf{T}+{ }^{t} \mathbf{J}-{ }^{t} \mathbf{J}_{0}\right)\right) \operatorname{det}\left(\mathbf{I}_{2 m}-u{ }^{t} \mathbf{J}_{0}\right) .
\end{gathered}
$$

But,

$$
\begin{aligned}
\operatorname{det}\left(\mathbf{I}_{2 m}-u{ }^{t} \mathbf{J}_{0}\right) & =\operatorname{det}\left(\left[\begin{array}{cc}
\mathbf{I}_{m} & u \mathbf{I}_{m} \\
\mathbf{0} & \mathbf{I}_{m}
\end{array}\right]\right) \operatorname{det}\left(\left[\begin{array}{cc}
\mathbf{I}_{m} & -u \mathbf{I}_{m} \\
-u \mathbf{I}_{m} & \mathbf{I}_{m}
\end{array}\right]\right) \\
& =\operatorname{det}\left(\left[\begin{array}{cc}
\left(1-u^{2}\right) \mathbf{I}_{m} & \mathbf{0} \\
* & \mathbf{I}_{m}
\end{array}\right]\right)=\left(1-u^{2}\right)^{m} .
\end{aligned}
$$


Therefore it follows that

$$
\left(1-u^{2}\right)^{2 m} \operatorname{det}\left(\mathbf{I}_{n}-u{ }^{t} \mathbf{W}_{x}(G)+\left(\mathbf{D}_{x}-\mathbf{I}_{n}\right) u^{2}\right)=\left(1-u^{2}\right)^{(m+n)} \operatorname{det}\left(\mathbf{I}_{2 m}-u\left({ }^{t} \mathbf{T}+{ }^{t} \mathbf{J}-{ }^{t} \mathbf{J}_{0}\right)\right) .
$$

Hence

$$
\left.\operatorname{det}\left(\mathbf{I}_{2 m}-u\left({ }^{t} \mathbf{B}-{ }^{t} \mathbf{J}_{0}\right)\right)=\left(1-u^{2}\right)^{(m-n)} \operatorname{det}\left(\mathbf{I}_{n}-u \mathbf{W}_{x}(G)+\left(\mathbf{D}_{x}-\mathbf{I}_{n}\right)\right) u^{2}\right)
$$

But, since

$$
i \mathbf{U}(\lambda)+\mathbf{J}_{0}={ }^{t} \mathbf{B}
$$

we have

$$
{ }^{t} \mathbf{B}-{ }^{t} \mathbf{J}_{0}=i \mathbf{U}(\lambda) .
$$

Substituting $u=-i$ in (11), we obtain

$$
\operatorname{det}\left(\mathbf{I}_{2 m}-\mathbf{U}(\lambda)\right)=2^{m-n} \operatorname{det}\left(\mathbf{I}_{n}+i \mathbf{W}_{x}(G)-\left(\mathbf{D}_{x}-\mathbf{I}_{n}\right)\right)
$$

By (4), (6) and (8), we have

$$
\mathbf{W}_{x}(G)={ }^{t} \mathbf{L K}={ }^{t} \mathbf{X}^{t} \mathbf{H K}=\mathbf{X} \tilde{\mathbf{C}}(G) .
$$

Furthermore, by (4), (7) and (9), we have

$$
\mathbf{D}_{x}={ }^{t} \mathbf{L H}={ }^{t} \mathbf{X}^{t} \mathbf{H H}=\mathbf{X} \tilde{\mathbf{D}} .
$$

Thus, we have

$$
\begin{gathered}
\operatorname{det}\left(\mathbf{I}_{2 m}-\mathbf{U}(\lambda)\right)=2^{m-n} \operatorname{det}\left(2 \mathbf{I}_{n}+\mathbf{X} \tilde{\mathbf{C}}(G)+i \mathbf{X} \tilde{\mathbf{D}}\right) \\
=2^{m-n} i^{n} \operatorname{det} \mathbf{X} \operatorname{det}\left(-2 i \mathbf{X}^{-1}+\tilde{\mathbf{C}}(G)+i \tilde{\mathbf{D}}\right)=\frac{2^{m} i^{n} \operatorname{det}\left(2 \mathbf{X}^{-1}+i \tilde{\mathbf{C}}(G)-\tilde{\mathbf{D}}\right)}{\prod_{j=1}^{n}\left(v_{j}-i v_{j}+\lambda i\right)} .
\end{gathered}
$$

Since $2 x_{j}^{-1}=v_{j}-i v_{j}+\lambda i$, we have

$$
-2 i \mathbf{X}^{-1}=-i(1-i) \tilde{\mathbf{D}}+\lambda \mathbf{I}_{n}
$$

and so

$$
-2 i \mathbf{X}^{-1}+\tilde{\mathbf{C}}(G)+i \tilde{\mathbf{D}}=\lambda \mathbf{I}_{n}+\tilde{\mathbf{C}}(G)-\tilde{\mathbf{D}}
$$

Hence

$$
\operatorname{det}\left(\mathbf{I}_{2 m}-\mathbf{U}(\lambda)\right)=\frac{2^{m} i^{n} \operatorname{det}\left(\lambda \mathbf{I}_{n}+\tilde{\mathbf{C}}(G)-\tilde{\mathbf{D}}\right)}{\prod_{j=1}^{n}\left(d_{j}-i d_{j}+\lambda i\right)}
$$

Q.E.D.

Let $G$ be a connected graph and $\tilde{\mathbf{C}}=\tilde{\mathbf{C}}(G)$ a weighted matrix of $G$. Then $G$ is called a $r$-regular weighted graph if $\sum_{o(b)=u} w(b)=r$ for each $u \in V(G)$.

By Theorem 7, the following result holds. 
Corollary 2 Let $G$ be an $r$-reguar weighted graph with $n$ vertices and $m$ edges, and $\tilde{\mathbf{C}}(G)$ a non-negative symmetric weighted matrix of $G$. Then

$$
\operatorname{det}\left(\mathbf{I}_{2 m}-\mathbf{U}(\lambda)\right)=2^{m} i^{n}(r-i r+\lambda i)^{-n} \operatorname{det}\left(\lambda \mathbf{I}_{n}+\tilde{\mathbf{C}}(G)-r \mathbf{I}_{n}\right) .
$$

Let $G=\left(V_{1}, V_{2}\right)$ be a bipartite graph. Then $G$ is called a $\left(q_{1}+1, q_{2}+1\right)$-semiregular weighted bipartite graph if $\sum_{o(e)=v} w(e)=q_{i}+1$ for each $v \in V_{i}(i=1,2)$.

Similarly to the proof of Theorem 6 , the following result holds.

Corollary 3 Let $G=\left(V_{1}, V_{2}\right)$ be a connected $\left(q_{1}+1, q_{2}+1\right)$-semiregular weighted bipartite graph with $\nu$ vertices and $\epsilon$ edges, and $\tilde{\mathbf{C}}=\tilde{\mathbf{C}}(G)$ a real symmetric weighted matrix of $G$. Set $\left|V_{1}\right|=n,\left|V_{2}\right|=m(n \leq m)$. Then

$\operatorname{det}\left(\mathbf{I}_{2 \epsilon}-\mathbf{U}(\lambda)\right)=2^{m} i^{n}\left(\lambda-q_{2}-1\right)^{m-n} \frac{\prod_{j=1}^{n}\left(\lambda^{2}-\left(q_{1}+q_{2}-2\right) \lambda+\left(q_{1}+1\right)\left(q_{2}+1\right)-\lambda_{j}^{2}\right)}{\left(\left(q_{1}+1\right)(1-i)+\lambda i\right)^{n}\left(\left(q_{2}+1\right)(1-i)+\lambda i\right)^{m}}$.

where $\operatorname{Spec}(\tilde{\mathbf{C}}(G))=\left\{ \pm \lambda_{1}, \cdots, \pm \lambda_{n}, 0, \cdots, 0\right\}$.

\section{The Euler product for a new zeta function}

We present the Euler product for a new zeta function of a graph.

Foata and Zeilberger [4] gave a new proof of Bass's Theorem by using the algebra of Lyndon words. Let $X$ be a finite nonempty set, $<$ a total order in $X$, and $X^{*}$ the free monoid generated by $X$. Then the total order $<$ on $X$ derive the lexicographic order $<$ on $X^{*}$. A Lyndon word in $X$ is defined to a nonempty word in $X^{*}$ which is prime, i.e., not the power $l^{r}$ of any other word $l$ for any $r \geq 2$, and which is also minimal in the class of its cyclic rearrangements under $<($ see $[9])$. Let $L$ denote the set of all Lyndon words in $X$.

Let $\mathbf{F}$ be a square matrix whose entries $b\left(x, x^{\prime}\right)\left(x, x^{\prime} \in X\right)$ form a set of commuting variables. If $w=x_{1} x_{2} \cdots x_{m}$ is a word in $X^{*}$, define

$$
\beta(w)=b\left(x_{1}, x_{2}\right) b\left(x_{2}, x_{3}\right) \cdots b\left(x_{m-1}, x_{m}\right) b\left(x_{m}, x_{1}\right) .
$$

Furthermore, let

$$
\beta(L)=\prod_{l \in L}(1-\beta(l))
$$

The following theorem played a central role in [4].

Theorem 8 (Foata and Zeilbereger) $\beta(L)=\operatorname{det}(\mathbf{I}-\mathbf{F})$.

Let $G$ be a connected graph and $\tilde{\mathbf{C}}(G)$ a weighted matrix of $G$. Then, let $w(e, f)$ be the $(e, f)$-array of the matrix $\mathbf{B}-\mathbf{J}_{0}$. 
Theorem 9 Let $G$ be a connected graph, and let $\tilde{\mathbf{C}}=\tilde{\mathbf{C}}(G)$ be a weighted matrix of $G$. Then the reciprocal of the zeta function of $G$ is given by

$$
\mathbf{Z}_{1}(G, w, t)=\prod_{[p]}\left(1-w_{p} t^{|p|}\right)^{-1}
$$

where $[p]$ runs over all primitive periodic orbits of $G$, and

$$
w_{p}=w\left(b_{1}, b_{2}\right) w\left(b_{2}, b_{3}\right) \cdots w\left(b_{n-1}, b_{n}\right), p=\left(b_{1}, b_{2}, \ldots, b_{n}\right)
$$

Proof. Let $R(G)=\left\{b_{1}, \cdots, b_{2 m}\right\}$ such that $b_{m+j}=\hat{b}_{j}(1 \leq j \leq m)$, and $b_{1}<b_{2}<$ $\cdots<b_{2 m}$ a total order of $R(G)$. We consider the free monid $R(G)^{*}$ generated by $R(G)$, and the lexicographic order on $R(G)^{*}$ derived from $<$. If a cycle $p$ is primitive, then there exists a unique cycle in $[p]$ which is a Lyndon word in $R(G)$.

For $z \in R(G)^{*}$, let

$$
\beta(z)= \begin{cases}w_{z} t^{|z|} & \text { if } z \text { is a primitive cycle } \\ 0 & \text { otherwise }\end{cases}
$$

Then we have

$$
\beta(L)=\prod_{l \in L}(1-\beta(l))=\prod_{[p]}\left(1-w_{p} t^{|p|}\right),
$$

where $[p]$ runs over all primitive periodic orbits of $G$. Furthermore, we define variables $b\left(x, x^{\prime}\right)\left(x, x^{\prime} \in R(G)\right)$ as follows:

$$
b\left(x, x^{\prime}\right)= \begin{cases}w\left(x, x^{\prime}\right) & \text { if } t(x)=o\left(x^{\prime}\right), \\ 0 & \text { otherwise. }\end{cases}
$$

Theorem 8 implies that

$$
\prod_{[p]}\left(1-w_{p} t^{|p|}\right)=\operatorname{det}(\mathbf{I}-t \mathbf{F})=\operatorname{det}\left(\mathbf{I}-t\left(\mathbf{B}-\mathbf{J}_{0}\right)\right) .
$$

Q.E.D.

\section{Acknowledgment}

This work is supported by Grant-in-Aid for Science Research (C) in Japan. We would like to thank the referee for valuable comments and helpful suggestions. 


\section{References}

[1] L. Bartholdi, Counting paths in graphs, Enseign. Math. 45 (1999), 83-131.

[2] H. Bass, The Ihara-Selberg zeta function of a tree lattice, Internat. J. Math. 3 (1992), 717-797.

[3] A. Comtet, J. Desbois and C. Texier, Functionals of the Brownian motion, localization and metric graphs, preprint [arXiv: cond-mat/0504513v2].

[4] D. Foata and D. Zeilberger, A combinatorial proof of Bass's evaluations of the IharaSelberg zeta function for graphs, Trans. Amer. Math. Soc. 351 (1999), 2257-2274.

[5] J. Desbois, Spectral determinant on graphs with generalized boundary conditions, Eur. Phys. J. B 24 (2001), 261-266.

[6] J. M. Harrison, U. Smilansky and B. Winn, Quantum graphs where back-scattering is prhibited, J. Phys. A:Math. Theor. 40 (2007), 14181-14193.

[7] K. Hashimoto, Zeta Functions of Finite Graphs and Representations of $p$-Adic Groups, in "Adv. Stud. Pure Math". Vol. 15, pp. 211-280, Academic Press, New York, 1989.

[8] Y. Ihara, On discrete subgroups of the two by two projective linear group over $p$-adic fields, J. Math. Soc. Japan 18 (1966), 219-235.

[9] M. Kotani and T. Sunada, Zeta functions of finite graphs, J. Math. Sci. U. Tokyo 7 (2000), 7-25.

[10] M. Lothaire, Combinatorics on Words, Addison-Wesley, Reading, Mass., 1983.

[11] I. Sato, A new zeta function of a graph, preprint.

[12] J. -P. Serre, Trees, Springer-Verlag, New York, 1980.

[13] U. Smilansky, Quantum chaos on discrete graphs, J. Phys. A: Math. Theor. 40 (2007), F621-F630.

[14] H. M. Stark and A. A. Terras, Zeta functions of finite graphs and coverings, Adv. Math. 121 (1996), 124-165.

[15] T. Sunada, L-Functions in Geometry and Some Applications, in "Lecture Notes in Math"., Vol. 1201, pp. 266-284, Springer-Verlag, New York, 1986.

[16] T. Sunada, Fundamental Groups and Laplacians(in Japanese), Kinokuniya, Tokyo, 1988. 